OPEN ACCESS

Edited by:

Elnara Shafiyeva,

Baku State University, Azerbaijan

Reviewed by:

Aleksandra Maria Rogowska,

Opole University, Poland

Cong Zhou,

Jining Medical University, China

${ }^{*}$ Correspondence: Minyoung Sim

minyoung.sim.yb@gmail.com

Specialty section:

This article was submitted to Public Mental Health, a section of the journal

Frontiers in Psychiatry

Received: 25 March 2021 Accepted: 31 May 2021

Published: 06 July 2021

Citation:

Lee JH, Lee D, Hyun S, Hong JS, Kim

C, Kim W and Sim M (2021) Online

Mental Health Assessments of COVID-19 Patients in South Korea.

Front. Psychiatry 12:685445.

doi: 10.3389/fpsyt.2021.685445

\section{Online Mental Health Assessments of COVID-19 Patients in South Korea}

\author{
Jung Hyun Lee, Dayoung Lee, Soyoen Hyun, Ji Sun Hong, Chang-Hoon Kim, Woojin Kim \\ and Minyoung Sim*
}

Division of Disaster Mental Health Services, National Center for Mental Health, Seoul, South Korea

Experiences of infectious diseases cause stressful and traumatic life events, hence, coronavirus disease 2019 (COVID-19) patients could suffer from various mental health problems requiring psychological support services. This study investigates the severity of mental health problems among confirmed COVID-19 patients. From March to November 2020, we collected the data from 118 COVID-19 patients who voluntarily participated in the National Center for Disaster Trauma's online mental health assessment consisting of self-report scales like Primary Care of Posttraumatic Stress Disorder screen (PC-PTSD), Generalized Anxiety Disorder-7 (GAD-7), Patient Health Questionnaire-9 (PHQ-9), Patient Health Questionnaire-15 (PHQ-15), and P4 Suicidality Screener. For control, 116 other disaster-experienced and 386 non-COVID-19-experienced participants were recruited. The COVID-19 patients showed more severe symptoms including post-traumatic symptoms, depression, anxiety, and somatic symptoms than control groups across all four screening scales $(p<0.001)$. Regarding high-risk, COVID-19 patients had an increased association with high-risk compared to the comparison groups (PC-PTSD: OR $=24.16,95 \% \mathrm{Cl}=13.52-43.16 p<0.001 ; \mathrm{PHQ}-9: \mathrm{OR}=14.45,95 \% \mathrm{Cl}=8.29-25.19$, $p<0.001 ; \mathrm{GAD}-7: \mathrm{OR}=20.71,95 \% \mathrm{Cl}=10.74-39.96, p<0.001 ; \mathrm{PHQ}-15: \mathrm{OR}=5.65$, $95 \% \mathrm{Cl}=3.44-9.25, p<0.001 ; \mathrm{P} 4: \mathrm{OR}=14.67,95 \% \mathrm{Cl}=8.95-25.07, p<0.001)$. This study's results imply that there is a high-risk of overall mental health problems, especially stronger associations of post-traumatic stress symptoms, in COVID-19 patients. These findings help inform practitioners about the psychological responses to COVID-19 experiences and to prepare appropriate interventions and services for the incremental number of confirmed cases.

Keywords: COVID-19, post-traumatic stress disorder, depression, anxiety, psychological trauma

\section{INTRODUCTION}

The coronavirus disease 2019 (COVID-19) outbreak is one of the largest pandemic disasters of this century. This disaster started in December 2019 and the World Health Organization (WHO) declared it a pandemic on $11^{\text {th }}$ March 2020 (1). An outbreak of COVID-19 has continued to worsen in Korea since the first confirmed patient was reported on January 2020. The need for mental health services during pandemics and other disasters was emphasized during the Middle East respiratory syndrome coronavirus (MERS-nCOV) outbreak in 2015, Therefore, multidisciplinary psychosocial support has been provided since the early stages of the outbreak in South Korea (2). The manifestation of COVID-19 varies from no symptoms to severe acute respiratory distress and high fatality (3). It is suggested that patients with COVID-19 may struggle with both, 
life-threatening fear of the infection and quarantine-related stressors that require psychological support during this pandemic $(4,5)$. However, there is little empirical evidence regarding COVID-19 survivors' mental health.

The outbreak of severe acute respiratory syndrome (SARS) gives us the most recent data on mental health problems related to infectious disease. Among SARS survivors who were discharged from the hospital for 4 weeks, about 32 and $27 \%$ of participants were classified as having over "moderate" ranges of anxiety and depressive symptoms, respectively, which were higher than community samples (6). Regarding the long-term psychiatric morbidities among SARS survivors, $25.6 \%$ of the patients had post-traumatic stress disorder (PTSD) and 15.6\% had depressive disorders 30 months after the SARS outbreak (7). A systematic review of coronavirus, including SARS, MERS-nCOV, and COVID-19, reported reduced health-related quality of life (HRQol) in survivors (8). Additionally, 38.8\% of them experienced post-traumatic stress disorder (PTSD), 33.2\%, depression, and 30.0\%, anxiety after 6 months (8). Previous studies suggested that a substantial portion of survivors suffered from psychiatric symptoms in either early or late aftermath of SARS. Besides physical symptoms, patients with infectious diseases experienced various stressors such as isolation, fear transmitting the virus to others, and social stigma which may lead them to experience psychological distress, loneliness, anxiety, depression, and post-traumatic stress symptoms (PTSS) $(5,9)$.

Most of the research related to COVID-19 and mental health has focused on the mental health of quarantined individuals and the general population. However, currently, there are only a few studies that have examined COVID-19 patients' mental health $(10,11)$. A study reported a high prevalence of PTSD and related risk factors in patients after severe COVID-19 (11). Furthermore, a recent retrospective cohort study reported increased risks of psychiatric and neurological morbidity in patients, six months after COVID-19 infection (12). Thus, an updated study on in COVID-19 patients' mental health problems is required to provide timely therapeutic approaches and mental health services. The National Center for Disaster Trauma (NCT) in South Korea was put in charge of psychological support services for COVID-19 patients. The NCT provides self-rated online assessments to encourage COVID-19 patients to use mental health services if needed. Self-rated assessments can provide valuable information to understand confirmed patients' psychological responses.

This study investigates confirmed COVID-19 patients' mental health, especially those who voluntarily seek mental health support. We hypothesized that COVID-19 patients would suffer from more severe symptoms including PTSS as well as depression, anxiety, somatic symptoms, and suicidality compared to those who did not have COVID-19.

\section{METHODS}

\section{Data Collection}

In Korea, the Integrated Psychological Support Group for COVID-19 under the Ministry of Health and Welfare has been set up to take charge of mental health services amid the COVID-19 outbreak from 29 $9^{\text {th }}$ January 2020 till date (2). National Center for Disaster Trauma (NCT) has a main role in managing this governmental organization and has provided psychological support service via a 24-h hotline for COVID-19 patients, quarantined individuals, and their families. To provide information, including stress management, available mental health services, and utility and online screening tool for mental health, we sent the text-messages to the list of confirmed patients given by the government. Additionally, individuals who visited either the official NCT website or social network services could also participate in the online mental health screening. When patients accessed the website, they were informed, "It is normal to feel any distress during or after COVID-19 treatment and quarantine, and it would be helpful to check one's psychological distress with the following online mental health assessment. Based on the result of the assessment, we will contact to you and provide psychological support services." Those who agreed and provided informed consent could start answering the questionnaires: demographic information (age and sex) and five self-reported scales. After completing the questionnaires they could find the total score for each test. From March to November 2020, 118 confirmed patients responded to the online mental health assessment, in this study.

As a comparison group, individuals who had not experienced COVID-19 were recruited and data from participants who participated in disaster mental health programs by the NCT, which provided regular education such as Psychological First Aid to the general public and mental health-related workers from 2018 to 2019, were used. Prior to the education, participants were asked to complete surveys, including the life-time experiences of disaster and mental health assessments, comprising the same scales as COVID-19 patients. A total of 492 participants voluntarily responded to the survey and were classified in disaster-experienced and -inexperienced groups in this study.

The present study was conducted as a part of the NCT research project, which was reviewed and approved by the Institutional Review Board of NCMH (approval No.116271-2020-29).

\section{Mental Health Assessment Tools}

The online mental health assessment comprised five screening scales including Primary Care of Posttraumatic Stress Disorder screen for DSM-5 (PC-PTSD), Generalized Anxiety Disorder7 (GAD-7), Patient Health Questionnaire-9 (PHQ-9), Patient Health Questionnaire-15 (PHQ-15), and P4 Suicidality Screener.

The PC-PTSD was used to assess the PTSS that were experienced during the last month $(13,14)$. Some of those in disaster-experienced and disaster-inexperienced group, who participated from 2018 to March 2019, completed the PCPTSD-4, meanwhile confirmed patients and those in the disaster-experienced group, who participated after March 2019, completed the PC-PTSD-5 (15). Participants answered "1: yes" or "0: no" for each item, and the severity of PTSS was classified based on the total score of each item such as 0-1: normal, 2: mild-severe and higher than 3: severe. Preliminary results from validation studies suggest a cut-point of 3 (13). PC-PTSD score was aggregated from 4 items which were common in PC-PTSD-4 and PC-PTSD-5. Based on the cut-off point of 3, we classified 
participants into "Above cut-off" and "Below cut-off" groups. Cronbach's alpha for 4 items used to aggregate PC-PTSD score was 0.76 in this study.

Patient Health Questionnaire (PHQ-9) was used to assess depression in the last 2 weeks (16). The PHQ-9 comprises nine items rated on 4-point Likert scale (0: Not at all-3: Nearly every day). The severity of depressive symptoms is classified into the following five groups: 0-4 (None), 5-9 (Mild), 10-14 (Moderate), 15-19 (Moderately severe), and over 20 (Severe); the suggested cutoff point is 10 . Participants with a total score over 10 were classified as the high-risk group and those with scores under 10, as the low-risk group. Cronbach's alpha for the PHQ-9 was 0.88 in this study.

Generalized Anxiety Disorder-7 (GAD-7) was used to assess anxiety symptoms in the previous 2 weeks (17). The GAD-7 comprises seven items rated on a 4-point Likert scale (0: Not at all-3: Nearly every day). The severity of the anxiety symptoms is classified into the four following groups: 0-4 (Normal), 59 (Mild), 10-14 (Moderate), and over 15 (Severe). A high-risk group comprised of those who received over 10 points (17). Cronbach's alpha for the GAD-7 was 0.89 in this study.

Patient Health Questionnaire-15 (PHQ-15) was used to assess somatic symptoms in the last month (18). The PHQ-15 consists of 15 items rated on 3-point Likert scale (0: Not bothered at all-2: Bothered a lot). The severity of somatic symptoms is classified into the following four groups: 0-4 (Normal), 5-9 (Mild), 10-14 (Moderate), and over 15 (Severe); the suggested cutoff point is 10. Participants with a total score over 15 were classified as the high-risk group. Cronbach's alpha for the PHQ-15 was 0.86 in this study.

The P4 Screener was used to assess potential suicide risk (19). It consists of four items: "(1) Have you ever attempted to harm yourself in the past?," "(2) Have you thought about how you might actually hurt yourself?," "(3) How likely do you think it is that you will act on these thoughts about hurting yourself or ending your life some time over the next month?", and "(4) Is there anything that would prevent or keep you from harming yourself?" If the answer is not "Yes" for the items (1) and (2), the participant is classified as at "Minimal" risk. If the participants chose "Somewhat likely" or "Very likely" for item (3), or "No" for item (4), they were classified as "Higher (high-risk group)," while the others were classified as "Lower (low-risk group)."

\section{Statistical Analysis}

To examine group differences among COVID-19 patients and disaster-experienced and -inexperienced individuals, demographic and clinical characteristics were analyzed using $\chi^{2}$ test for categorical variables and analysis of variance (ANOVA) for continuous variables. Comparison of each mental health assessment was performed using ANOVA with Tukey's HSD post-hoc test. We perform a Pearson correlation analysis to determine the statistic relationship between each of the mentalhealth-related variables. Additionally, multivariate logistic regression was used to explore the association among COVID-19 patients' moderate to severe symptoms and the comparison groups. The "Above cut-off" group of each scale was used as the outcome variable. Then, the odds ratio for the "Above cut-off" group among COVID-19 patients was calculated using covariates, including age and sex. The significance level was set at $p<0.05$. Statistical analyses were conducted using the statistical package $\mathrm{R}$ version 4.0 .2 for Windows.

TABLE 2 | Pearson correlation matrix for mental-health-related variables in COVID-19 patients $(n=118)$.

\begin{tabular}{lcccr}
\hline Variables & $\mathbf{1}$ & $\mathbf{2}$ & $\mathbf{3}$ & $\mathbf{4}$ \\
\hline 1. PC-PTSD & 1 & - & - & - \\
2. PHQ-9 & $0.67^{\star}$ & 1 & - & - \\
3. GAD-7 & $0.59^{\star}$ & $0.86^{\star}$ & 1 & - \\
4. PHQ-15 & $0.44^{\star}$ & $0.68^{\star}$ & $0.65^{\star}$ & 1 \\
\hline
\end{tabular}

PC-PCSD, Primary Care PTSD Screen; PHQ-9, Patient Health Questionnaire-9; GAD-7, Generalized Anxiety Disorder; Screener P4, P4 suicidality Screener. ${ }^{\star} p<0.01$.

TABLE 1 | Demographic and clinical characteristics of the participants.

\begin{tabular}{|c|c|c|c|c|c|c|}
\hline & $\begin{array}{l}\text { COVID-19 Patients (A) } \\
(N=118)\end{array}$ & $\begin{array}{l}\text { Disaster-experienced (B) } \\
\qquad(N=116)\end{array}$ & $\begin{array}{l}\text { Disaster-inexperienced (C) } \\
\qquad(N=386)\end{array}$ & $X^{2} / F$ & Post-hoc Tukey HSD & $p$ \\
\hline Age & $32.69 \pm 13.59$ & $41.04 \pm 16.15$ & $43.88 \pm 10.67$ & $F_{(2,605)}=36.08$ & $\mathrm{~A}<\mathrm{B}, \mathrm{A}<\mathrm{C}$ & $<0.001$ \\
\hline Sex & & & & $X_{(2)}^{2}=15.04$ & & \\
\hline Males & 34 (28.8\%) & $28(24.3 \%)$ & 159 (41.8\%) & & & $<0.001$ \\
\hline Females & 84 (71.2\%) & 87 (75.7\%) & 221 (58.2\%) & & & \\
\hline PC-PTSD & $2.5 \pm 1.4$ & $1.0 \pm 1.3$ & $0.7 \pm 1.1$ & $F_{(2,604)}=102.40$ & $A>B>C$ & $<0.001$ \\
\hline PHQ9 & $11.21 \pm 6.68$ & $4.50 \pm 5.32$ & $4.03 \pm 4.04$ & $F_{(2,604)}=99.36$ & $A>B, A>C$ & $<0.001$ \\
\hline GAD & $9.24 \pm 5.60$ & $2.85 \pm 3.86$ & $2.40 \pm 3.06$ & $F_{(2,583)}=147.90$ & $A>B, A>C$ & $<0.001$ \\
\hline PHQ15 & $10.53 \pm 5.57$ & $6.59 \pm 5.40$ & $5.16 \pm 4.47$ & $F_{(2,605)}=53.98$ & $A>B>C$ & $<0.001$ \\
\hline \multicolumn{7}{|l|}{ P4 } \\
\hline Low risk & 106 (89.8\%) & 110 (98.2\%) & 376 (99.5\%) & $X_{(2)}^{2}=33.00$ & $A>B>C$ & $<0.001$ \\
\hline High risk & $12(10.2 \%)$ & $2(1.8 \%)$ & $2(0.5 \%)$ & & & \\
\hline
\end{tabular}

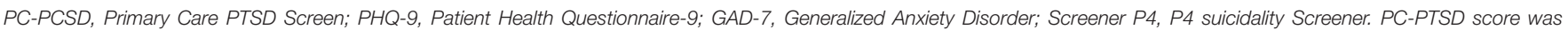
calculated by summing the 4 items that were common in PC-PTSD-4 and PC-PTSD-5. 


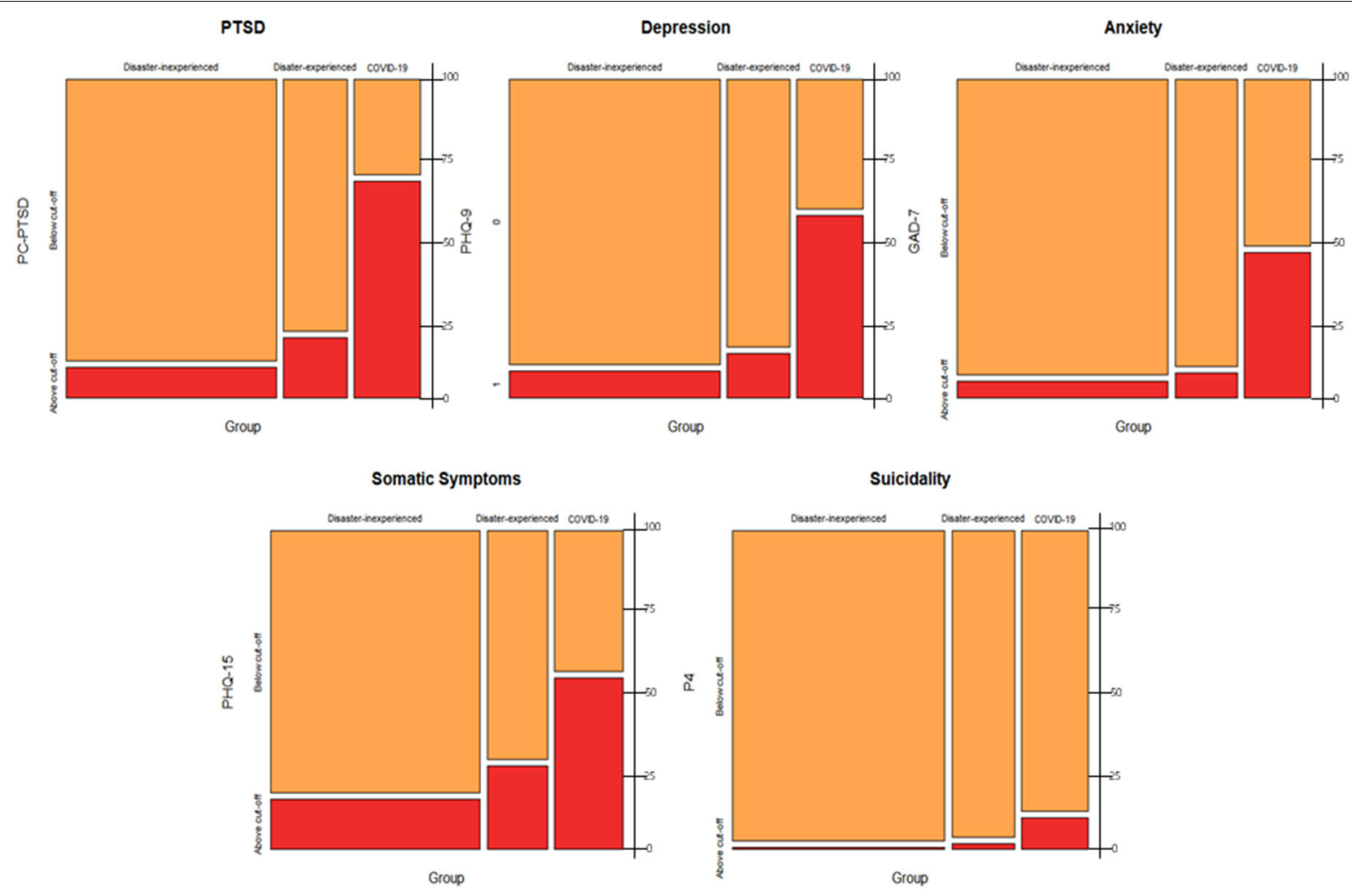

FIGURE 1 | Mosaic plots of severe mental health problems among COVID-19 patients and comparison groups. Mosaic plot depicting that the proportion of "above cut-off" group (red) for each of the mental-health-related variables in COVID-19 patients is significantly greater than that in the control groups. PC-PCSD, Primary Care PTSD Screen; PHQ-9, Patient Health Questionnaire-9; GAD-7, Generalized Anxiety Disorder; Screener P4, P4 suicidality Screener.

\section{RESULTS}

A total of 118 COVID-19 patients participated in the online mental health assessment. Of which, 34 (28.8\%) were male and $84(71.4 \%)$ were female. The mean age of COVID-19 patients in the online mental health assessment was 32.69 ( $\mathrm{SD}=13.59)$, which was lower than that of the disaster-experienced and inexperienced groups $\left\{F_{(2,605)}=36.08, p<0.001\right\}$. The result of ANOVA indicated that there were statistically significant differences in all mental health assessment scores $\{$ (PC-PTSD, $F_{(2,604)}=102.4, p<0.001$; PHQ-9: $F_{(2,604)}=99.36, p<0.001$; GAD-7, $F_{(2,605)}=147.9, p<0.001$; PHQ-15: $F_{(2,53.98)}=59.98$, $p<0.001)$ In the post-hoc analysis, COVID-19 patients showed higher total score in all measurements compared to the other groups (Table 1). Among the COVID-19 patients, the mental health assessments were significantly correlated with each other $(p<0.001)$, with correlation coefficients ranging from 0.44 to 0.86 (Table 2). The PHQ-9 was the most strongly correlated with the GAD-7 ( $r=0.86)$; it was also strongly correlated with the PC-PTSD and the PHQ-9 ( $r=0.67$ and $r=0.68$, respectively). There were moderate correlations between the PC-PTSD and the GAD-7 and the PC-PTSD and the PHQ-15 ( $r=0.59$ and $r=$ 0.44 , respectively).

The proportions of "Above cut-off" for each assessment were significantly different in COVID-19 patients and disaster-experienced and -inexperienced groups (Figure 1, PC-PTSD, $\chi_{(2)}^{2}=123.75, p<0.001$; PHQ-9, $\chi_{(2)}^{2}=88.78, p<$ 0.001 ; GAD-7, $\chi_{(2)}^{2}=160.42, p<0.001$; PHQ-15, $\chi_{(2)}^{2}=53.96$, $p<0.001)$.

The result of logistic regression suggested that, COVID-19 patients had higher adjusted odds ratios (AOR) for "Above cutoff" across all measurements compared to disaster-inexperienced group (PC-PTSD, AOR = 24.14, 95\% CI = 13.52-43.16; PHQ-9, $\mathrm{AOR}=14.45,95 \% \mathrm{CI}=8.29-25.19 ; \mathrm{GAD}-7, \mathrm{AOR}=20.71,95 \%$ $\mathrm{CI}=10.74-39.96$; PHQ-15, $\mathrm{AOR}=5.65,95 \% \mathrm{CI}=3.44-9.25 ; \mathrm{P} 4$, $\mathrm{AOR}=14.67,95 \% \mathrm{CI}=8.95-25.07)$. The COVID-19 patients had stronger associations with significant AORs for PC-PTSD and PHQ-15 compared to disaster-experience group (Table 3).

\section{DISCUSSION}

This study provides empirical data on mental health problems related to COVID-19. We found that more than half the COVID-19 patients who participated in the online mental health assessment experienced post-traumatic stress, depression, anxiety, and somatic symptoms. Additionally, one in ten was in the high-risk group for suicidality. There is a paucity of knowledge regarding COVID-19's impact on mental health. Until now, despite the increased concerns about COVID-19 
TABLE 3 | Adjusted odds ratio of severe mental health problems among COVID-19 patients and comparison groups.

\begin{tabular}{|c|c|c|c|c|c|c|}
\hline & \multicolumn{2}{|c|}{$\begin{array}{c}\text { Disaster- } \\
\text { inexperienced } \\
\text { Group }(n=386)\end{array}$} & \multicolumn{2}{|c|}{$\begin{array}{c}\text { Disaster- } \\
\text { experienced } \\
\text { Group }(n=116)\end{array}$} & \multicolumn{2}{|c|}{$\begin{array}{l}\text { COVID-19 patients } \\
(n=118)\end{array}$} \\
\hline & $n(\%)$ & $\begin{array}{c}\text { OR } \\
(95 \% \mathrm{Cl})\end{array}$ & $n(\%)$ & $\begin{array}{c}\text { OR } \\
(95 \% \mathrm{Cl})\end{array}$ & $n(\%)$ & $\begin{array}{c}\text { OR } \\
(95 \% \mathrm{Cl})\end{array}$ \\
\hline \multicolumn{7}{|l|}{ PC-PTSD } \\
\hline $\begin{array}{l}\text { Below } \\
\text { cut-off }\end{array}$ & $\begin{array}{c}339 \\
(90.2 \%)\end{array}$ & & $\begin{array}{c}92 \\
(80.7 \%)\end{array}$ & & $\begin{array}{c}36 \\
(30.5 \%)\end{array}$ & \\
\hline $\begin{array}{l}\text { Above } \\
\text { cut-off }\end{array}$ & $\begin{array}{c}37 \\
(9.8 \%)\end{array}$ & 1.0 & $\begin{array}{c}22 \\
(19.3 \%)\end{array}$ & $\begin{array}{c}2.02 \\
(1.11-3.67)^{\star}\end{array}$ & $\begin{array}{c}82 \\
(69.5 \%)\end{array}$ & $\begin{array}{c}24.16 \\
(13.52-43.16)^{\star \star}\end{array}$ \\
\hline \multicolumn{7}{|l|}{ PHQ-9 } \\
\hline $\begin{array}{l}\text { Below } \\
\text { cut-off }\end{array}$ & $\begin{array}{c}344 \\
(91.2 \%)\end{array}$ & & $\begin{array}{c}96 \\
(85.7 \%)\end{array}$ & & $\begin{array}{c}49 \\
(41.5 \%)\end{array}$ & \\
\hline $\begin{array}{l}\text { Above } \\
\text { cut-off }\end{array}$ & $\begin{array}{c}33 \\
(8.8 \%)\end{array}$ & 1.0 & $\begin{array}{c}16 \\
(14.3 \%)\end{array}$ & $\begin{array}{c}1.71 \\
(0.89-3.27)\end{array}$ & $\begin{array}{c}69 \\
(58.5 \%)\end{array}$ & $\begin{array}{c}14.45 \\
(8.29-25.19)^{\star \star}\end{array}$ \\
\hline \multicolumn{7}{|l|}{ GAD-7 } \\
\hline $\begin{array}{l}\text { Below } \\
\text { cut-off }\end{array}$ & $\begin{array}{c}358 \\
(94.7 \%)\end{array}$ & & $\begin{array}{c}103 \\
(92.0 \%)\end{array}$ & & $\begin{array}{c}63 \\
(53.4 \%)\end{array}$ & \\
\hline $\begin{array}{l}\text { Above } \\
\text { cut-off }\end{array}$ & $\begin{array}{c}20 \\
(5.3 \%)\end{array}$ & 1.0 & $\begin{array}{c}9 \\
(8.0 \%)\end{array}$ & $\begin{array}{c}1.65 \\
(0.72-3.80)\end{array}$ & $\begin{array}{c}55 \\
(46.6 \%)\end{array}$ & $\begin{array}{c}20.71 \\
(10.74-39.96)^{\star \star}\end{array}$ \\
\hline \multicolumn{7}{|l|}{ PHQ-15 } \\
\hline $\begin{array}{l}\text { Below } \\
\text { cut-off }\end{array}$ & $\begin{array}{c}305 \\
(84.0 \%)\end{array}$ & & $\begin{array}{c}77 \\
(73.3 \%)\end{array}$ & & $\begin{array}{c}53 \\
(44.9 \%)\end{array}$ & \\
\hline $\begin{array}{l}\text { Above } \\
\text { cut-off }\end{array}$ & $\begin{array}{c}58 \\
(16.0 \%)\end{array}$ & 1.0 & $\begin{array}{c}28 \\
(26.7 \%)\end{array}$ & $\begin{array}{c}1.73 \\
(1.02-2.94)^{*}\end{array}$ & $\begin{array}{c}65 \\
(55.1 \%)\end{array}$ & $\begin{array}{c}5.65 \\
(3.44-9.25)^{\star \star}\end{array}$ \\
\hline \multicolumn{7}{|l|}{ P4 } \\
\hline $\begin{array}{l}\text { Below } \\
\text { cut-off }\end{array}$ & $\begin{array}{c}376 \\
(99.5 \%)\end{array}$ & & $\begin{array}{c}110 \\
(98.2 \%)\end{array}$ & & $\begin{array}{c}106 \\
(89.8 \%)\end{array}$ & \\
\hline $\begin{array}{l}\text { Above } \\
\text { cut-off }\end{array}$ & $\begin{array}{c}2 \\
(0.5 \%)\end{array}$ & 1.0 & $\begin{array}{c}2 \\
(1.8 \%)\end{array}$ & $\begin{array}{c}1.11 \\
(0.64-1.93)\end{array}$ & $\begin{array}{c}12 \\
(10.2 \%)\end{array}$ & $\begin{array}{c}14.67 \\
(8.95-25.07)^{\star \star}\end{array}$ \\
\hline
\end{tabular}

PC-PCSD, Primary Care PTSD Screen; PHQ-9, Patient Health Questionnaire-9; GAD-7, Generalized Anxiety Disorder; Screener P4, P4 suicidality Screener.

Odds ratios were adjusted for covariates including age and sex.

${ }^{\star} p<0.05,{ }^{* \star} p<0.001$

infection as a stressful event, research that evaluates the mental health problems among COVID-19 patients is scarce. In our study, COVID-19 patients reported more severe symptoms including PTSS, depression, anxiety, somatic symptoms, and suicidality compared to the comparison groups who never experienced COVID-19. Additionally, compared to other disaster-experienced individuals from the comparison group, COVID-19 patients received higher scores on all mental health assessments. The "Above cut-off" group, showed greater odds ratios PTSS, depression, somatic symptoms, and suicidality in COVID-19 patients compared to the disaster-inexperienced group. Remarkably, these associations were stronger than those in the disaster-experienced group.

Consistent with the results of previous large-scale pandemic studies, we found that COVID-19 patients have significantly higher rates of mental health problems than the control groups. Existing studies report an alarming proportion $(\sim 40 \%)$ of SARS or MERS survivors having experienced psychiatric illnesses $(14,20)$. A long-term follow-up study reported that $45 \%$ of participants experienced at least one more psychiatric disorder event after discharge and 58.9\% cumulative incidence of psychiatric disorder in the post-SARS era (7). The evidence found in this study points to a strong association between COVID-19 and adverse mental health issues, compared with the control groups. This is in agreement with a previous finding of increased hazard ratios of anxiety and mood disorders in COVID-19 survivors $(12,21)$. PTSD is commonly seen in those who have survived disasters $(22,23)$. In our sample, PTSS were most common, with more than two thirds of the COVID-19 patients reporting an "above cut-off" score on the PC-PTSD scale. Additionally, the odds ratio of PTSS was greater than that of other mental health problems in the COVID-19 patients. The likelihood of severe PTSS was 24 times higher in the COVID-19 patients group compared to the control group, which is twofold higher than the odds ratio of other disaster-experienced groups. Consistent with the definition of traumatic events as exposure to death, threatened death, actual or threatened serious injury, the experience of COVID-19 infection may be sufficient to cause life-threatening fear and helplessness (24). Patients with infectious diseases are likely to experience post-traumatic stress responses due to the traumatic course of infection, including treatment, quarantine, and social stigma (25). Former reports with a high prevalence of PTSD among SARS survivors have been supporting our results. Mak et al. (7) reported that about half of the patients experienced PTSD after the SARS outbreak, and $25 \%$ of the patients had been diagnosed with PTSD through clinical interviews even 30-months post-SARS; half of them had a PTSD diagnosis after the SARS outbreak. A study, conducted in China, reported that over 95\% of COVID-19 patients who completed an online assessment were found to have PTSS (10). These rates are notably higher than those of SARS survivors. Through a meta-analysis study it was found that the prevalence of PTSD was $32.2 \%$, which was two times higher than depression and anxiety disorder, in the post-illness stage among COVID-19 patients (23). The most recent study, which used the ClinicallyAdministered PTSD Scale for COVID-19 patients, found that that the prevalence of PTSD was $30.2 \%$, which is greater than previous reports of other types of disasters, such as earthquakes or the World Trade Center disaster (11). Regarding traumarelated symptoms, $75 \%$ of Ebola survivors reported re-experience or arousal (26). Since the PC-PTSD is a screening tool for PTSD, clinical diagnosis cannot be awarded for scores above the cut-off; the figures in our sample may have been exaggerated compared to those in previous studies. However, our findings imply that a more individuals may have suffered from PTSS after COVID-19 infection compared to other disasters, which is consistent with a previous study (11). It is therefore essential to provide timely management to alleviate PTSS for COVID-19 patients.

Regarding depression and anxiety, approximately half our participants were classified into higher cut-off groups and had a greater likelihood of mental health issues, compared to the control groups. Previous reports of SARS showed that $10-40 \%$ of patients reported over moderate levels of anxiety and depression (7), in the immediate aftermath of SARS. A recent survey on the general population's mental health after the COVID-19 pandemic in South Korea showed that 20.0 and $16.3 \%$ of participants scored above the cut-off scores on PHQ-9 and GAD-7, respectively (27). 
Although direct comparison between our study's findings and with these data is limited, the proportions of confirmed patients in the "Above cut-off" groups, in our study, was greater, with 58.5\% for PHQ-9 and 46.6\% for GAD-7.

Interestingly, somatic symptoms were also more frequent in COVID-19 patients than in the control group. Somatic symptoms might be directly related to COVID-19 infection or occur because of psychological distress. Trauma or stressors can impair the autonomic nervous system or the stress response system (28), causing somatization or non-specific physical symptoms. Physical symptoms, such as pain, fatigue, and general weakness, make it harder for respiratory infection survivors to return to their work and normal life (29-31). Post-SARS patients complained of fatigue, myalgia, and weakness accompanied by depression and sleep disturbances (30). Vittori et al. (32) suggest a multidisciplinary approach to identify physical and psychological disabilities and long-term effects in survivors of COVD-19.

Holmes et al. (33) suggested that strong and specific stressors to COVID-19 infection could have a profound effect on the mental health of COVID-19 patients. Consistent with mental health experts' primary concern, a considerable number of COVID-19 patients in our study were potentially at a high-risk for mental health problems. Despite the urgent need for mental health research, psychiatric symptoms in COVID-19 patients have not been investigated using validated screening tools (33). Furthermore, most previous studies related to infectious diseases have used retrospective recall methods to assess survivors, however, our data represented the current psychological response to COVID-19. Our findings highlight the need for psychosocial support for the infected patients. Additionally, initial screening for high-risk or vulnerable individuals would be helpful to mitigate long-term adverse consequences. Due to the crosssectional design and limited information regarding the COVID19 patients' medical history, our results could not confirm causalrelationship between COVID-19 and mental health problems. However, COVID-19 experiences can constitute a traumatic event beyond a mere stressor. Furthermore, COVID-19 invades the central nervous system, impacting the individual's mental health $(34,35)$.

There are several limitations to this study. First, as the number of subjects in the dataset is relatively small compared to the total number of confirmed patients in the country, our results may not comprehensively reflect all COVID-19 patients. Additionally, selective biases may have occurred as our sample may have included more individuals who were worried about their mental health problems or those who tend to seek the required help. Additionally, this online assessment collected data only from those who have access to computers or smartphones, therefore, the surveys were responded to by younger participants in the COVID-19 group. Hence, the odds ratio in our analysis cannot be generalized to all the COVID19 patients. Second, multiple factors could be associated with poorer mental health, however, we only had little information regarding demographic and treatment-related factors. The time interval between the disaster event and mental health assessment, which may affect the severity of mental health symptoms, was not collected in our study. Heterogeneity in time intervals might be greater in the control group than in the COVID-19 group.
Additionally, we could not gather sufficient information, such as duration of treatment/quarantine, past psychiatry history, and psychosocial stressors, because our data were collected to provide psychological support to those who needed help, rather than for research purposes. As individuals who experience infectious disease tend to be hesitant to disclose their personal information due to fear of social stigma, minimal demographic and clinical information was collected in the online assessments in this study. Conversely, anonymity would be helpful for screening in communities as this may help confirmed patients respond more honestly.

Despite of these limitations, we carefully emphasize that our findings can provide valuable information regarding the severity of mental health symptoms among COVID-19 patients. This study highlights the need to enhance preparedness regarding mental health support to better manage COVID-19 patients' psychological responses amid the COVID-19 pandemic. Future research should include longitudinal follow-up studies of COVID-19 patients to explore the long-term psychiatric consequences and related risk and protective factors.

\section{DATA AVAILABILITY STATEMENT}

Derived data supporting the findings of this study are available from the corresponding author on request.

\section{ETHICS STATEMENT}

The studies involving human participants were reviewed and approved by Institutional Review Board of NCMH. Written informed consent for participation was not required for this study in accordance with the national legislation and the institutional requirements.

\section{AUTHOR CONTRIBUTIONS}

JL and MS devised the project, the main conceptual ideas, and proof outline. $\mathrm{SH}, \mathrm{JH}, \mathrm{C}-\mathrm{HK}$, and $\mathrm{WK}$ contributed to data collection and preparation. SH and JL analyzed data. JL, DL, and MS contributed to the interpretation of the results. JL took the lead in writing the manuscript. All authors provided critical feedback and helped shape the research, analysis, and manuscript.

\section{FUNDING}

This study was supported by a clinical research grant (No. 2021-09) from the National Center for Mental Health, Republic of Korea.

\section{ACKNOWLEDGMENTS}

We gratefully acknowledge the support the National Center for Trauma provided for this study. We would like to thank the members of the Integrated Psychological Support Group for COVID-19, Republic of Korea. 


\section{REFERENCES}

1. World Health Organization. Coronavirus Disease 2019 (COVID-19) Situation Report - 51. New York, NY: World Health Organization (2020). Available online at: https://www.who.int/docs/default-source/coronaviruse/situationreports/20200311-sitrep-51-covid-19.pdf?sfvrsn=1ba62e57_10 (accessed January 20, 2021).

2. Hyun J, You S, Sohn S, Kim S-J, Bae J, Baik M, et al. Psychosocial support during the COVID-19 outbreak in Korea: activities of multidisciplinary mental health professionals. J Korean Med Sci. (2020) 35:e211. doi: 10.3346/jkms.2020.35.e211

3. Huang C, Wang Y, Li X, Ren L, Zhao J, Hu Y, et al. Clinical features of patients infected with 2019 novel coronavirus in Wuhan, China. Lancet. (2020) 395:497-506. doi: 10.1016/S0140-6736(20)3 0183-5

4. Park SC, Park YC. Mental health care measures in response to the 2019 novel coronavirus outbreak in Korea. Psyc Investig. (2020) 17:856. doi: $10.30773 /$ pi.2020.0058

5. Xiang YT, Yang Y, Li W, Zhang L, Zhang Q, Cheung T, et al. Timely mental health care for the 2019 novel coronavirus outbreak is urgently needed. Lancet Psychiatry. (2020) 7:228-29. doi: 10.1016/S2215-0366(20)3 0046-8

6. Cheng SK, Wong CW, Tsang J, Wong KC. Psychological distress and negative appraisals in survivors of severe acute respiratory syndrome (SARS). Psychol Med. (2004) 34:1187--95. doi: 10.1017/S00332917040 02272

7. Mak IW, Chu CM, Pan PC, Yiu MG, Chan VL. Long-term psychiatric morbidities among SARS survivors. Gen Hosp Psychiatry. (2009) 31:31826. doi: 10.1016/j.genhosppsych.2009.03.001

8. Ahmed H, Patel K, Greenwood DC, Halpin S, Lewthwaite P, Salawu $\mathrm{A}$, et al. Long-term clinical outcomes in survivors of severe acute respiratory syndrome and Middle East respiratory syndrome coronavirus outbreaks after hospitalisation or ICU admission: a systematic review and meta-analysis. J Rehabil Med. (2020) 52:jrm00063. doi: 10.2340/1650197 7-2694

9. Wu KK, Chan SK, Ma TM. Posttraumatic stress, anxiety, and depression in survivors of severe acute respiratory syndrome (SARS). J Trauma Stress. (2005) 18:39-42. doi: 10.1002/jts.20004

10. Bo HX, Li W, Yang Y, Wang Y, Zhang Q, Cheung T, et al. Posttraumatic stress symptoms and attitude toward crisis mental health services among clinically stable patients with COVID-19 in China. Psychol Med. (2020) 51:1052-3-. doi: 10.1017/S0033291720000999

11. Janiri D, Carfi A, Kotzalidis GD, Bernabei R, Landi F, Sani G, et al. Posttraumatic stress disorder in patients after severe COVID-19 infection. JAMA Psychiatry. (2021) 78:567-9. doi: 10.1001/jamapsychiatry.20 21.0109

12. Taquet M, Geddes JR, Husain M, Luciano S, Harrison PJ. 6-month neurological and psychiatric outcomes in 236379 survivors of COVID19: a retrospective cohort study using electronic health records. Lancet Psychiatry. (2021) 8:416-27. doi: 10.1016/S2215-0366(21)0 0084-5

13. Cameron RP, Gusman D. The primary care PTSD screen (PC-PTSD): development and operating characteristics. Prim Care Psychiatry. (2003) 9:9-14. doi: 10.1185/135525703125002360

14. Lam MH, Wing YK, Yu MW, Leung CM, Ma RC, Kong AP, et al. Mental morbidities and chronic fatigue in severe acute respiratory syndrome survivors: long-term follow-up. Arch Intern Med. (2009) 169:214247. doi: 10.1001/archinternmed.2009.384

15. Prins A, Bovin MJ, Smolenski DJ, Marx BP, Kimerling R, JenkinsGuarnieri MA, et al. The primary care PTSD screen for DSM-5 (PCPTSD-5): development and evaluation within a veteran primary care sample. J Gen Intern Med. (2016) 31:1206-11. doi: 10.1007/s11606-01 6-3703-5

16. Spitzer RL, Kroenke K, Williams JB. Validation and utility of a self-report version of PRIME-MD: the PHQ primary care study. JAMA. (1999) 282:173744. doi: 10.1001/jama.282.18.1737
17. Spitzer RL, Kroenke K, Williams JB, Lowe B. A brief measure for assessing generalized anxiety disorder: the GAD-7. Arch Intern Med. (2006) 166:109297. doi: 10.1001/archinte.166.10.1092

18. Kroenke K, Spitzer RL, Williams JB. The PHQ-15: validity of a new measure for evaluating the severity of somatic symptoms. Psychosom Med. (2002) 64:258-66. doi: 10.1097/00006842-200203000-00008

19. Dube P, Kurt K, Bair MJ, Theobald D, Williams LS. The p4 screener: evaluation of a brief measure for assessing potential suicide risk in 2 randomized effectiveness trials of primary care and oncology patients. Prim Care Companion J Clin Psychiatry. (2010) 12:12. doi: 10.4088/PCC.10m00 978blu

20. Kim HC, Yoo SY, Lee BH, Lee SH, Shin HS. Psychiatric findings in suspected and confirmed Middle East respiratory syndrome patients quarantined in hospital: a retrospective chart analysis. Psychiatry Investig. (2018) 15:35560. doi: 10.30773/pi.2017.10.25.1

21. Taquet M, Luciano S, Geddes JR, Harrison PJ. Bidirectional associations between COVID-19 and psychiatric disorder: retrospective cohort studies of 62354 COVID-19 cases in the USA. Lancet Psychiatry. (2021) 8:13040. doi: 10.1016/S2215-0366(20)30462-4

22. Perrin MA, DiGrande L, Wheeler K, Thorpe L, Farfel M, Brackbill R. Differences in PTSD prevalence and associated risk factors among World Trade Center disaster rescue and recovery workers. Am J Psychiatry. (2007) 164:1385-94. doi: 10.1176/appi.ajp.2007.061 01645

23. Rogers JP, Chesney E, Oliver D, Pollak TA, McGuire P, Fusar-Poli $\mathrm{P}$, et al. Psychiatric and neuropsychiatric presentations associated with severe coronavirus infections: a systematic review and meta-analysis with comparison to the COVID-19 pandemic. Lancet Psychiatry. (2020) 7:61127. doi: 10.1016/S2215-0366(20)30203-0

24. American Psychiatric Association. Diagnostic and Statistical Manual of Mental Disorders (DSM-5®). Arlington, VA: American Psychiatric Association (2013). p. 5-25.

25. Van Bortel T, Basnayake A, Wurie F, Jambai M, Koroma AS, Muana AT, et al. Psychosocial effects of an Ebola outbreak at individual, community and international levels. Bull World Health Organ. (2016) 94:21014. doi: 10.2471/BLT.15.158543

26. Hugo M, Declerck H, Fitzpatrick G, Severy N, Gbabai OB-M, Decroo $\mathrm{T}$, et al. Post-traumatic stress reactions in Ebola virus disease survivors in Sierra Leone. Emerg Med. (2015) 5:1-4. doi: 10.4172/2165-7548.10 00285

27. Korean Ministry of Health and Welfare: Society of Traumatic Stress. The 4th Mental Health Survey During COVID-19. (2021). Available online at: http:// kstss.kr/wp-content/uploads/2021/01/KSTSS21-01-18-COVID-19-\%EA \%B5\%AD\%EB\%AF\%BC\%EC\%A0\%95\%EC\%8B\%A0\%ЕA\%B1\%B4\%EA\%B0 \%95\%ЕC\%8B\%A4\%ED\%83\%9C\%EC\%A1\%B0\%EC\%82\%AC_4\%EC\%B0 \%A8.pdf. (accessed 15 May, 2021)

28. Fries E, Hesse J, Hellhammer J, Hellhammer DH. A new view on hypocortisolism. Psychoneuroendocrinology. (2005) 30:101016. doi: 10.1016/j.psyneuen.2005.04.006

29. Herridge MS, Cheung AM, Tansey C.M, Matte-Martyn A, DiazGranados N, Al-Saidi F, et al. One-year outcomes in survivors of the acute respiratory distress syndrome. N Engl J Med. (2003) 348:683-93. doi: 10.1056/NEJMoa022450

30. Moldofsky H, Patcai J. Chronic widespread musculoskeletal pain, fatigue, depression and disordered sleep in chronic post-SARS syndrome; a case-controlled study. BMC Neurol. (2011) 11:37. doi: 10.1186/1471-237 7-11-37

31. Kamdar BB, Sepulveda KA, Chong A, Lord RK, Dinglas VD, MendezTellez PA, et al. Return to work and lost earnings after acute respiratory distress syndrome: a 5-year prospective, longitudinal study of longterm survivors. Thorax. (2018) 73:125-33. doi: 10.1136/thoraxjnl-2017-2 10217

32. Vittori A, Lerman J, Cascella M, Gomez-Morad AD, Marchetti G, Marinangeli F, et al. COVID-19 pandemic acute respitory distress syndrome survivors: pain after the Storm? Anesth Analg. (2020) 31:117-19. doi: 10.1213/ANE.0000000000004914 
33. Holmes EA, O'Connor RC, Perry VH, Tracey I, Wessely S, Arseneault L, et al. Multidisciplinary research priorities for the COVID-19 pandemic: a call for action for mental health science. Lancet Psychiatry. (2020) 7:54760. doi: 10.1016/S2215-0366(20)30168-1

34. He L, Ding YQ, Che XY, Zhang QL, Huang ZX, Wang HJ, et al. Expression of the monoclonal antibody against nucleocapsid antigen of SARS-associated coronavirus in autopsy tissues from SARS patients. Di Yi Jun Yi Da Xue Xue Bao. (2003) 23:1128-30.

35. Lu W, Wang H, Lin Y, Li L. Psychological status of medical workforce during the COVID-19 pandemic: a cross-sectional study. Psychiatry Res. (2020) 288:112936. doi: 10.1016/j.psychres.2020.112936
Conflict of Interest: The authors declare that the research was conducted in the absence of any commercial or financial relationships that could be construed as a potential conflict of interest.

Copyright (c) 2021 Lee, Lee, Hyun, Hong, Kim, Kim and Sim. This is an open-access article distributed under the terms of the Creative Commons Attribution License (CC $B Y)$. The use, distribution or reproduction in other forums is permitted, provided the original author(s) and the copyright owner(s) are credited and that the original publication in this journal is cited, in accordance with accepted academic practice. No use, distribution or reproduction is permitted which does not comply with these terms. 